\title{
Anesthetic Management in the Case of Premature Infant with Bronchopulmonary Dysplasia and Retinopathy of Prematurity
}

\author{
Demet Coskun ${ }^{\mathrm{a}, \mathrm{c}}$, Ahmet Mahlia ${ }^{\mathrm{a}}$, Tolga Tezer ${ }^{\mathrm{a}}$, Sengul Ozdek ${ }^{\mathrm{b}}$
}

\begin{abstract}
In this case report, we present the anesthetic management towards an infant with atelectasis of the right lung, and with bronchopulmonary dysplasia (BPD), for whom laser treatment is planned for retinopathy of prematurity (ROP). This female infant weighing 950 grams and born through normal vaginal delivery at the 27 th gestational age was transferred to our hospital following the diagnosis of BPD and ROP. Standard monitorization was performed for the patient and sevoflurane induction with $\mathrm{O}_{2}$ was administered, and a laryngeal mask airway (LMA) was placed. One minute later, the patient's peripheric oxygen saturation $\left(\mathrm{SpO}_{2}\right)$ decreased to $70 \%$. Considering that the increased secretion of the patient should be aspirated, endotracheal intubation was performed. Concordantly, it was observed that the $\mathrm{SpO}_{2}$ of the patient reached $100 \%$ and this value was maintained until the end of the surgery. We conclude that, when administering general anesthesia to these patients, instead of the placement of a LMA, the direct endotracheal intubation would be more efficient to ensure a patent airway and easily aspirate secretions.
\end{abstract}

Keywords: Anesthesia; Retinopathy of prematurity; Bronchopulmonary dysplasia

\section{Introduction}

It is well known that so many complications of prematurity

\footnotetext{
Manuscript accepted for publication May 20, 2010

${ }^{a}$ Department of Anaesthesiology and Reanimation, Gazi University Faculty of Medicine, Ankara, Turkey

${ }^{\mathrm{b}}$ Department of Ophthalmology, Gazi University Faculty of Medicine, Ankara, Turkey

${ }^{c}$ corresponding author: Gazi University Faculty of Medicine, Department of Anaesthesiology and Reanimation, 06500-ANKARA, Turkey. Email: dcoskun@gazi.edu.tr
}

can develop in the premature infants. Some of these complications may require surgical interventions [1]. In addition, premature infants are at a greater risk than the full term infants while undergoing similar surgery $[2,3]$. Because of the fact that almost all premature infants have various degrees of bronchopulmonary dysplasia (BPD), the risk of encountering the symptoms of coughing, laryngospasm and consequent hypoxic episodes is getting higher in the postoperative period [4].

In this case report, we present the anesthetic management towards an infant with a complete atelectasis of the right lung, and with bronchopulmonary dysplasia, for whom laser treatment is planned for rethinopathy of prematurity (ROP).

\section{Case Report}

The female infant weighing 950 grams and born through vaginal delivery at the 27th gestational age was transferred to our hospital following the diagnosis of BPD and consequent bilateral stage 2 retinopathy with plus disease. As a result of the examinations and tests performed, the patient was hospitalized following the diagnosis of prematurity, respiratory distress syndrome, sepsis, BPD, ROP, transient hypothyroxinemia of prematurity and intracranial hemorrhage. During the physical examination of the patient, tachypnea and retractions were observed. In the transfontanel ultrasonography performed, it was observed that both ventricles were dilated, thrombosis was present in choroid plexus, and heterogenous image in the left thalamus was seen and cystical encephalomalacia was present. In the echocardiography, secundum atrial septal defect, minimal tricuspid regurgitation, and patent ductus arteriosus were observed. When an urgent ROP surgery was planned for the patient with the above mentioned symptoms, during the preoperative assessment in terms of anesthesia, the chest radiogram showed a complete atelectasis of the right lung (Fig. 1).

The peripheric oxygen saturation $\left(\mathrm{SpO}_{2}\right)$ of the infant, who was admitted to the operation room in an incubator with oxygen support, was observed to be $94 \%$. Standard monitorization was performed for the patient and ventilation 


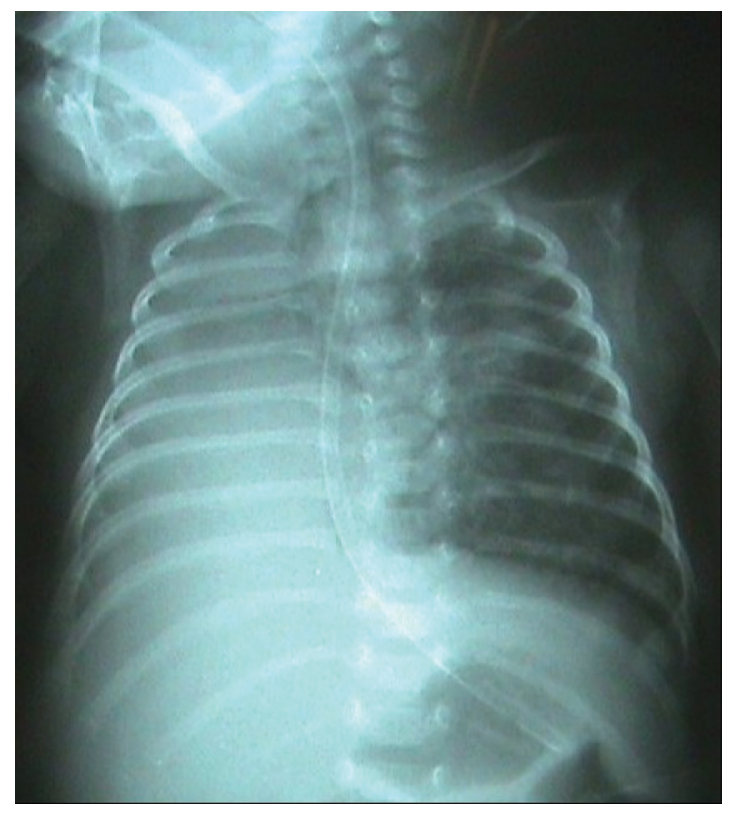

Figure 1. Chest radiogram preoperatively.

was manually assisted by a face mask, and the $\mathrm{SpO}_{2}$ was found to be $97 \%$. Following this, sevoflurane induction with $\mathrm{O}_{2}$ was administered, and a laringeal mask airway (LMA) was placed. One minute later, the patient's $\mathrm{SpO}_{2}$ decreased to $70 \%$. The LMA was immediately removed and the patient was ventilated by a face mask. The $\mathrm{SpO}_{2}$ thus reached 97\%. Then, the LMA was placed once again and the $\mathrm{SpO}_{2}$ again decreased to $70 \%$. The LMA was again removed and the patient was ventilated by a face mask. Then the patient was intubated with an endotracheal tube No. 2.5. However, when desaturation continued, the tube was removed and

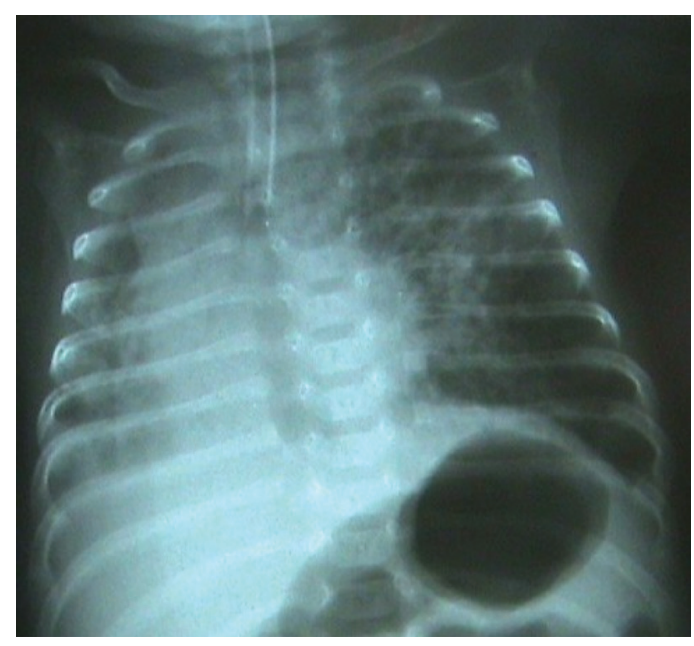

Figure 2. Chest radiogram in the 2 nd postoperative hour.

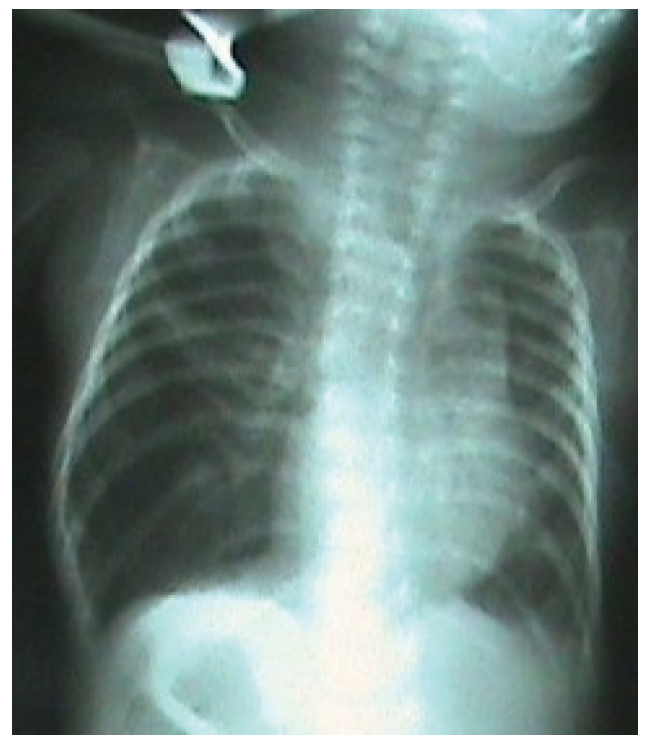

Figure 3. Chest radiogram on the 1st postoperative day.

again, the patient was ventilated by a face mask. The $\mathrm{SpO}_{2}$ of the infant rapidly increased to $96 \%$. It was observed that the removed tube was obstructed with secretion. The patient was intubated a second time with an endotracheal tube No. 3.0 and a large amount of secretion was aspirated. While the patient was being ventilated with a mixture of $40 \% \mathrm{O}_{2}$-air, the $\mathrm{SpO}_{2}$ improved gradually and reached $100 \%$. This value was maintained during the 30-minute surgery. Laser treatment was applied to both eyes of the patient and the surgery ended without any complications. At the end of the surgery, the intubated patient was transferred to the pediatric intensive care unit. The clinically stable patient was extubated after the confirmation of the $\mathrm{SpO}_{2}$ to be $100 \%$ at the 2 nd postoperative hour and right lung, which had atelectasis in the chest radiogram, to be significantly treated (Fig. 2). In the chest radiogram of the patient, who was followed up in the pediatric intensive care unit, taken the following day, it was observed that the right lung was normally ventilated (Fig. 3). Thus, the patient was transferred to the maternity ward.

\section{Discussion}

Bronchopulmonary dysplasia is the most common cause of chronic lung disease in infants [5]. Rather than being a specific disease, it is the symptom of a complex one characterized by airway obstruction, airway hyperreactivity, and lung hyperinflation [4]. This results in uneven distribution of ventilation, reduced compliance, increased work of breathing, and compromised gas exchange. The clinical manifestations of this condition are rapid respiration, wheezing, cough, and 
frequent episodes of fever, desaturation, and bradycardia [1, 4]. Instrumentation of the airway is associated with pulmonary, as well as systemic, hypertension [6]. Further respiratory compromise may prove to be disastrous in these patients, and it is well documented that infants with BPD are at increased risk to develop bronchospasm, atelectasis, and pneumonia in the postoperative period $[2,7]$.

The early detection of stage 3 ROP is crucial for the exact timing of the treatment [8]. When treatment is delayed, development of retinal detachment and subsequent very low visual acuity or blindness is more likely [9]. Therefore, in our case, the infant with atelectasis of a single lung was immediately admitted for surgery without waiting for the recovery of the lung.

There is great variation on the types of anesthesia for the surgery of ROP including general anesthesia, topical anesthesia alone or topical anesthesia plus oral sedation or intravenous sedation or rectal chloralhydrate or paracetamol or intravenous ketamine, subtenon's local anesthesia, ketamine sedation, remifentanyl infusion and morphine infusion [1015]. However, when we look through the literature, all anesthesia types have their own advantages and disadvantages on the risk of morbidity and the patient's, surgeon's and the anesthetist's security, so the anesthesia technique to be used must be chosen according to the patient's clinical aspect.

Topical anesthesia alone is insufficient for treatment of ROP and may be associated with an increased incidence of potentially life-threatening cardio-respiratory events. Among the variety of anesthesia alternatives for surgery of ROP, general anesthesia is the mostly used modality. General anesthesia in premature infants can be associated with a high risk of morbidity especially when intraventricular hemorrhage, patent ductus arteriosus, and necrotising enterocolitis are also present $[10,11,16]$. In this case report; because the patient had an atelectesia on the right lung and low the $\mathrm{SpO}_{2}$ (94\%) although she was in the incubator with the $\mathrm{O}_{2}$ supply, we decided to choose general anesthesia technique where we can use positive pressure ventilation.

Patients with ROP and concomitant BPD present the anesthesiologist with a significant challenge regarding airway management. General anesthesia with tracheal intubation will guarantee a patent airway, make positive pressure ventilation possible, and allow the anesthesiologist to remove their hand from the surgical field. However, these patients are sensitive to airway infections and often suffer from bronchial hyperreactivity [17]. Thus, inserting a foreign object into the bronchial tree will increase the risk both for respiratory infection and bronchospastic episodes. General anesthesia and tracheal intubation also is not uncommonly associated with a setback regarding the need for respiratory support, and certain patients thus will require postoperative ventilator treatment of varying duration [14, 18, 19].

It is possible to administer general anesthesia by a face mask, however, when a face mask is used, the mask or the hand of the anesthesiologist holding the mask may cause a failure in the successful completion of the surgery. Furthermore, pressure from the mask and the hand may cause an increase in intraocular pressure on the globe [19]. The incidence of coughing, straining movements, breath holding, and sore throat were much higher when the endotrachael tube was used when compared to the use of LMA. Since LMA does not enter the trachea, it is less irritating to both the upper and lower airways; therefore, it may be advantageous in former premature infants and other children with chronic lung disease [20]. Also, in pediatric patients, the LMA does not stimulate the sensitive tissue of the larynx and trachea. Therefore, when laryngeal stimulation is absent, for minor surgical procedures, pediatric patients may be maintained at a lighter plane of general anesthesia [19]. So, we decided to use the LMA at the beginning of the operation but as the patient's $\mathrm{SpO}_{2}$ was insistently low, we had to intubate the infant with an endotrachael tube.

In conclusion, even if premature patients with BPD also have atelectasis of one lung, such patients should immediately be operated on for such surgeries are urgent. When administering general anesthesia to these patients, instead of the placement of LMA, we believe direct endotracheal intubation would be more efficient to ensure a patent airway and easily aspirate secretions.

\section{References}

1. Bhutani VK, Abbasi S. Long-term pulmonary consequences in survivors with bronchopulmonary dysplasia. Clin Perinatol 1992;19(3):649-671.

2. Steward DJ. Preterm infants are more prone to complications following minor surgery than are term infants. Anesthesiology 1982;56(4):304-306.

3. Liu LM, Cote CJ, Goudsouzian NG, Ryan JF, Firestone S, Dedrick DF, Liu PL, et al. Life-threatening apnea in infants recovering from anesthesia. Anesthesiology 1983;59(6):506-510.

4. Vasallo SA, Goudsouzian NG. Bronchopulmonary dysplasia. Curr Pract Anesthesiol 1990;2:222-226.

5. Northway WH, Jr. An introduction to bronchopulmonary dysplasia. Clin Perinatol 1992;19(3):489-495.

6. Hickey PR, Hansen DD, Wessel DL, Lang P, Jonas RA, Elixson EM. Blunting of stress responses in the pulmonary circulation of infants by fentanyl. Anesth Analg 1985;64(12):1137-1142.

7. Spear RM. Anesthesia for premature and term infants: perioperative implications. J Pediatr 1992;120(2 Pt 1):165-176.

8. Hindle NW. Cryotherapy for retinopathy of prematurity: timing of intervention. Br J Ophthalmol 1986;70(4):269276.

9. Reynolds JD, Hardy RJ, Kennedy KA, Spencer R, van 
Heuven WA, Fielder AR. Lack of efficacy of light reduction in preventing retinopathy of prematurity. Light Reduction in Retinopathy of Prematurity (LIGHT-ROP) Cooperative Group. N Engl J Med 1998;338(22):15721576.

10. Chen SD, Sundaram V, Wilkinson A, Patel CK. Variation in anaesthesia for the laser treatment of retinopathy of prematurity--a survey of ophthalmologists in the UK. Eye (Lond) 2007;21(8):1033-1036.

11. Parulekar MV, Chen SD, Patel CK. Sub-Tenon's local anaesthesia for the treatment of retinopathy of prematurity with diode laser. Eye (Lond) 2008;22(3):375-379.

12. Lyon F, Dabbs T, O'Meara M. Ketamine sedation during the treatment of retinopathy of prematurity. Eye (Lond) 2008;22(5):684-686.

13. Haigh PM, Chiswick ML, O'Donoghue EP. Retinopathy of prematurity: systemic complications associated with different anaesthetic techniques at treatment. Br J Ophthalmol 1997;81(4):283-287.

14. Kirwan C, O'Keefe M, Prendergast M, Twomey A, Murphy J. Morphine analgesia as an alternative to general anaesthesia during laser treatment of retinopathy of prematurity. Acta Ophthalmol Scand 2007;85(6):644-
647.

15. Sammartino M, Bocci MG, Ferro G, Mercurio G, Papacci P, Conti G, Lepore D, et al. Efficacy and safety of continuous intravenous infusion of remifentanil in preterm infants undergoing laser therapy in retinopathy of prematurity: clinical experience. Paediatr Anaesth 2003;13(7):596-602.

16. Welborn LG, Greenspun JC. Anesthesia and apnea. Perioperative considerations in the former preterm infant. Pediatr Clin North Am 1994;41(1):181-198.

17. Northway WH, Jr. Bronchopulmonary dysplasia: then and now. Arch Dis Child 1990;65(10 Spec No):10761081.

18. Lonnqvist PA. Successful use of laryngeal mask airway in low-weight expremature infants with bronchopulmonary dysplasia undergoing cryotherapy for retinopathy of the premature. Anesthesiology 1995;83(2):422-424.

19. Ferrari LR, Goudsouzian NG. The use of the laryngeal mask airway in children with bronchopulmonary dysplasia. Anesth Analg 1995;81(2):310-313.

20. Johnston DF, Wrigley SR, Robb PJ, Jones HE. The laryngeal mask airway in paediatric anaesthesia. Anaesthesia 1990;45(11):924-927. 